\title{
AVALIAÇÃO DOS NÍVEIS DE INTENSIDADE DE ESFORÇO DURANTE O JOGO DE RUGBY EM CADEIRAS DE RODAS
}

\author{
Renata Santos ${ }^{1}$ \\ Luís Gustavo de Souza Pena² \\ Mauro Furtado de Souza ${ }^{3}$ \\ Jessica Reis Buratti ${ }^{4}$ \\ Rafael Ribeiro Mattosinho ${ }^{5}$ \\ Fernando Rosch de Faria ${ }^{6}$ \\ José Irineu Gorla ${ }^{7}$
}

SANTOS, R.; PENA, L. G. de S.; SOUZA, M. F. de; BURATTI, J. R.; MATTOSINHO, R. R.; FARIA, F. R. de; GORLA, J. I. Avaliação dos níveis de intensidade de esforço durante o jogo de rugby em cadeiras de rodas . Arq. Cienc. Saúde UNIPAR, Umuarama, v. 22, n. 3, p, 145-149, set./dez. 2018.

RESUMO: O Rugby em cadeira de rodas (RCR) é um esporte paralímpico praticado por homens e mulheres com tetraplegia decorrente de lesão na medula espinhal (LME). Nos esportes de alto rendimento o desempenho do atleta depende de preparo físico, aspectos tático e técnico. Dentre as variáveis fisiológicas importantes no controle do treinamento e na resposta aos treinos encontra-se a frequência cardíaca (FC). O presente estudo teve como objetivo verificar a intensidade da FC durante jogos de RCR. Para isso, utilizou-se do frequencímetro FIRTSBEAT modelo SPORTS Team 4.6 ${ }^{\circledR}$, para o monitoramento da FC dos atletas de RCR durante os jogos. A amostra foi composta por nove atletas homens de RCR com LME com nível de lesão acima da vértebra T6. Os resultados obtidos demonstraram que a média das FC máximas foi de 138,55 \pm 17,5 bpm. A intensidade variou cerca de 77\% do tempo entre Zona aeróbia 1, Zona aeróbia 2 e Zona de limiar anaeróbio, indicando uma grande oscilação, o que é esperado por se tratar de um esporte que possui características de intermitência. Conclui-se que os atletas de RCR apresentaram uma FC máxima reduzida, correspondente às encontradas na literatura, e que esses apresentaram uma variação da intensidade da FC, com predominância entre $60 \%$ a $90 \%$ da FC máxima.

PALAVRAS-CHAVE: Frequência cardíaca. Pessoas com deficiência. Rugby.

\section{EFFORT INTENSITY LEVELS DURING A WHEELCHAIR RUGBY GAME}

\begin{abstract}
Wheelchair Rugby (WR) is a Paralympic sport modality practiced by men and women with spinal cord injury (SCI). In high performance sports, the performance of the athlete depends on his physical fitness, as well as tactical and technical aspects. Heart rate (HR) features among the several essential physiological variables in the control of the training and in response to training. This study aimed to determine the HR intensity during WR matches. In order to do so, FIRSTBEAT SPORTS Team 4.6® heart rate monitors were used to monitor the HR of the WR athletes during the games. The sample consisted of nine male WR athletes with SCI with impairment level above T6. The results showed that the average maximum HR was $138 \pm 17.5 \mathrm{bpm}$. Intensity varied in approximately $77 \%$ of the time between the Aerobic Zone 1, Aerobic Zone 2 and the Anaerobic Threshold Zone, presenting great oscillation. This is expected, since rugby is a sport with intermittent characteristics. It was concluded that WR athletes showed reduced HR (max), similar to the results found in literature, and presented variation in the HR intensity, with a predominance between $60 \%$ to $90 \%$ of the HR(max).
\end{abstract}

KEYWORDS: Heart rate. Paralimpic sport. Wheelchair rugby.

\section{Introdução}

Com os avanços da tecnologia no esporte de alto rendimento, novas metodologias e aprimoramento profissional por parte dos técnicos e equipe técnica em relação aos aspectos táticos e técnicos da modalidade, o Rugby em Cadeira de Rodas (RCR) vem ganhando adeptos e ascensão a cada ano. O RCR foi criado em 1977 no Canadá, destinado à atletas com tetraplegia ou lesões semelhantes em termos de comprometimento, seu surgimento partiu da observação de uma dificuldade apresentada pelos atletas em jogar o então já existente Basquete em Cadeira de Rodas (IWRF, 2016).

Segundo a Federação Internacional de Rugby em Cadeira de Rodas (IWRF), o RCR em 1994, foi reconhecido oficialmente como esporte paralímpico e teve um campeonato mundial nos anos seguintes, mas foi em 2000, em Sydnei, que o RCR foi disputado pela primeira vez em uma paralimpíada. Atualmente, 29 países estão listados no ranking internacional (IWRF, 2016).

Uma das ferramentas de controle em treinamentos e jogos, é o monitoramento da Frequência Cardíaca (FC). A partir do Monitorando da FC é possível analisar a intensidade de esforço e com isso, identificar se o atleta está em sua frequência máxima ou se poderá se esforçar mais nos treinos e competições e ainda, se deve diminuir o ritmo, sob risco de comprometer sua saúde.

Sendo os atletas praticantes da modalidade necessariamente indivíduos com tetraplegia, tendo sofrido lesão na medula espinhal (LME), sabe-se que gera perda do sistema simpático, resultando em uma atenuação da frequência cardíaca máxima (FC max.), além de prejudicar a regulação do retorno venoso e do volume sistólico promovendo débi-

DOI: 10.25110/arqsaude.v22i3.2018.6403

${ }^{1} A v$. Érico Veríssimo, 701, Barão Geraldo-Campinas-SP. E-mail: renta_msantos1995@hotmail.com

${ }^{2}$ Av. Érico Veríssimo, 701, Barão Geraldo-Campinas-SP. E-mail: pena_fef06@yahoo.com.br

${ }^{3}$ Av. Érico Veríssimo, 701, Barão Geraldo-Campinas-SP. E-mail: mauro_furtados@hotmail.com

${ }^{4}$ Av. Érico Veríssimo, 701, Barão Geraldo- Campinas-SP. E-mail: jerburatti@gmail.com

${ }^{5}$ Rua Capivari 569, Bairro Jardim Campos Elizios - Campinas-Sp. E-mail: rafaelmattosinho@yahoo.com.br

${ }^{6}$ Av. Érico Veríssimo, 701, Barão Geraldo- Campinas-SP. E-mail: nandorosch@yahoo.com.br

${ }^{7}$ Av. Érico Veríssimo, 701, Barão Geraldo- Campinas-SP. E-mail: gorla@fef.unicamp.br 
to cardíaco, sendo tais disfunções autonômicas dependentes do nível da lesão do individuo (KRASSIOUKOV; WEST, 2014). Outra questão importante é o fato da pessoa com lesão medular possuir uma fadiga precoce (PELLETIER; HICKS, 2011), sendo que alguns dos fatores responsáveis por isso são a menor mobilidade do diafragma e o não controle da musculatura torácica. Também, devido à sua disfunção autonômica, o atleta de RCR possui um descontrole em sua homeostase de modo geral (MYERS, LEE; KIRATLI, 2007).

Devido à tetraplegia, em geral as pessoas com LME têm grande dificuldade em encontrar uma prática esportiva a qual se adequem bem (NASH, 2005). Nesse sentido observa-se que os baixos níveis de atividade física, conjuntamente com o perfil lipídico, advindo ou não da primeira condição, contribuem para um maior risco de doenças cardiovasculares em pessoas com LME (LAVIS; SCELZA; BOCKENEK, 2007), sendo tais doenças apontadas como algumas das maiores responsáveis pela mortalidade dessas pessoas (MYERS; LEE; KIRATLI, 2007).

Apesar das pessoas com LME possuírem uma resposta diminuída ao exercício físico, além de uma maior dificuldade na manutenção da temperatura corporal (NASH, 2005), é recomendado que elas pratiquem alguma atividade física. Um estudo que avaliou por meio de uma revisão bibliográfica exercícios para pacientes com LME, chegou à conclusão de que a prática regular de exercícios melhoram a qualidade de vida desses indivíduos, e ainda que a ausência dessa prática pode levar a uma diminuição de sua funcionalidade e o aumento de doenças cardiovasculares (DE-
VILLARD et al., 2007).

Sendo o RCR um esporte coletivo de impacto, tendo sido pensada e criada especificamente para indivíduos com tetraplegia, compreender as questões fisiológicas do atleta em uma partida é de fundamental importância, visto que no jogo temos momentos de esforço físico intenso e momentos de esforço diminuído, tendo esses acontecimento influência sobre a saúde e performance. Dessa forma, o presente estudo teve como objetivo verificar a intensidade da FC durante jogos de RCR.

\section{Método}

De acordo com Thomas, Nelson e Silverman (2007), este estudo caracteriza-se como pesquisa descritiva e apresenta um delineamento transversal. A população deste estudo foi composta por nove atletas com LME, a nível cervical, do sexo masculino, praticantes de RCR. Os atletas pertencem ao projeto de extensão da Universidade Estadual de Campinas (UNICAMP), competem pela Associação de Desportos Adaptados de Campinas (ADEACAMP). A amostra foi escolhida devido a sua grande representatividade em nível nacional, sendo a equipe tetra campeã brasileira e possuindo quatro atletas da seleção brasileira de RCR no ano de 2016. Realizou-se o monitoramento no total de treze jogos, não sendo necessariamente o mesmo atleta monitorado em todos os jogos. A Tabela 1 apresenta a descrição dos sujeitos da pesquisa.

Tabela 1: Dados da amostra.

\begin{tabular}{c|c|c|c|c}
\hline Sujeitos & Idade (anos). & $\begin{array}{c}\text { Experiência no } \\
\text { Rugby em cadeira de } \\
\text { rodas (anos). }\end{array}$ & $\begin{array}{c}\text { Carga horária Semanal } \\
\text { em Rugby em cadeira de } \\
\text { rodas (horas). }\end{array}$ & Tempo de Lesão (anos). \\
\hline 1 & 28 & 8 & 12 & 11 \\
\hline 2 & 29 & 8 & 6 & 9 \\
\hline 3 & 40 & 5 & 9 & 12 \\
\hline 4 & 28 & 1 & 6 & 13 \\
\hline 5 & 29 & 7 & 12 & 13 \\
\hline 6 & 36 & 6 & 12 & 12 \\
\hline 7 & 38 & 8 & 15 & 6 \\
\hline 8 & 27 & 6 & 12 & 11 \\
\hline 9 & 30 & 4 & 10,67 & 11 \\
\hline Média & 31,67 & 5,89 & 3,04 & 3,74 \\
\hline
\end{tabular}

Pra inclusão no estudo, optou-se por atletas que tivessem uma carga horária de treinamento sistematizada de no mínimo seis horas semanais, ou seja, classificados como frequentes na escala de atividade física (WASHBURN et al., 2002); voluntários do sexo masculino; praticantes da modalidade há pelo menos um ano e com experiência em pelo menos uma competição. Para uma maior confiabilidade nas informações da intensidade de esforço, foram excluídos da análise dos dados os atletas que não apresentaram participação de, no mínimo, 1/3 do período de tempo de jogo.

O presente trabalho foi aprovado pelo Comitê de Ética em Pesquisa com Seres Humanos, da Faculdade de Ci- ências Medicas, da Universidade Estadual de Campinas, sob o numero 83875/2012.

Para o desenvolvimento deste estudo, a aferição dos batimentos cardíacos foi realizada por meio do aparelho de marca FIRSTBEAT modelo SPORTS Team 4.6®. Firstbeat é um software de uso profissional em testes esportivos, treinamento e análise da performance de equipes e atletas individualmente. O método é baseado em uma análise avançada dos dados da frequência e variabilidade cardíaca, propiciando o monitoramento em tempo real da carga de treinamento, fornecendo dados do \%FC máx, \%VO2 máx, EPOC, TRIMP e gasto energético. O equipamento é composto pela cinta 
peitoral (capaz de refletir a atividade do sistema nervoso autônomo) e por uma antena receptora que ao ser acoplado ao computador com o software transmite os dados instantaneamente da cinta peitoral para o computador.

Para a determinação das zonas de intensidade o software realiza um cálculo levando em consideração a FC máxima de cada atleta, definindo 5 zonas: Recuperação - 50$60 \%$ da FC máx, Aeróbio $1-60-70 \%$ da FC máx, Aeróbio $2-70-80 \%$ da FC máx, Limiar Anaeróbio - 80-90\% da FC máx, e Alta Intensidade - 90-100\% da FC máx, o alocamento nas zonas é realizado através da computação do tempo em que o atleta permance com a FC nas referidas zonas durante o período de registro. Para a identificação da frequência cardíaca máxima, o software realiza atualização constante considerando a maior frequência cardíaca apresentada pelo atleta durante todas as coletas realizadas.

Para a realização da coleta foi definido o seguinte protocolo. Ao chegarem ao local do jogo, os atletas foram equipados com a cinta peitoral e foi verificado se o sinal do aparelho estava funcionando corretamente e possíveis ajustes foram realizados. Após o processo de certificação do aparelho, a frequência cardíaca foi monitorada desde o aquecimento pré-jogo e durante toda a partida, sendo registrada também durante a recuperação devido às substituições realizadas. Em algumas situações o aquecimento pré-jogo não foi monitorado em razão de um jogo ser realizado após o outro.

Após a coleta, os dados de todos os jogos foram extraídos e tabulados em média, desvio padrão e percentual, através do pacote Microsoft Excel 2007®.

\section{Resultados}

A média das frequências cardíacas máximas dos atletas durante os jogos foi de 138,55 \pm 17,5 batimentos por minuto (bpm). Sendo a média das frequências cardíacas médias dos atletas durante os jogos de 108,70 $\pm 14,45 \mathrm{bpm}$.

Sobre a intensidade de esforço, os dados apontaram que durante os jogo os atletas permaneceram cerca de $77 \%$ do tempo total da coleta entre as zonas de límear anaeróbio, zona aeróbia 2 e zona aeróbia 1 (Figura 1).

Figura 1: Intensidade de esforço durante o jogo de Rugby em cadeira de rodas.

Zonas de Intensidade durante o jogo de Rugby em cadeira de rodas.

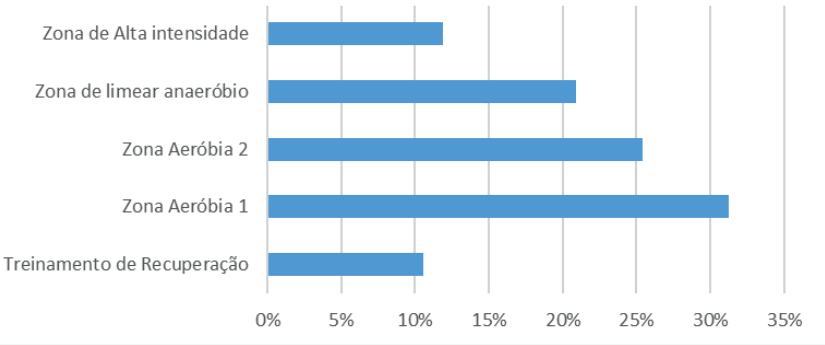

Os dados individuais relacionados às zonas de intensidade dos atletas durante a competição são apresentados na tablea 2 .

Tabela 2: Intensidade de esforço do jogo de Rugby em cadeira de rodas subdividido por atletas.

\begin{tabular}{c|c|c|c|c|c|c}
\hline SUJEITOS & C. F & RECUP. & AER. 1 & AER. 2 & LIMIAR ANAE. & ALTA INT. \\
\hline 1 & 2,5 & $1,61 \%$ & $13,65 \%$ & $24,50 \%$ & $40,96 \%$ & $19,28 \%$ \\
\hline 2 & 2,5 & $16,41 \%$ & $27,81 \%$ & $22,04 \%$ & $27,05 \%$ & $6,69 \%$ \\
\hline 3 & 2,5 & $15,49 \%$ & $48,36 \%$ & $14,55 \%$ & $12,21 \%$ & $9,39 \%$ \\
\hline 4 & 2 & $0,29 \%$ & $33,92 \%$ & $49,42 \%$ & $14,04 \%$ & $2,34 \%$ \\
\hline 5 & 2 & $25,34 \%$ & $29,56 \%$ & $34,88 \%$ & $8,31 \%$ & $1,91 \%$ \\
\hline 6 & 2 & $3,20 \%$ & $48,86 \%$ & $16,44 \%$ & $23,29 \%$ & $8,22 \%$ \\
\hline 7 & 1 & $13,11 \%$ & $33,98 \%$ & $17,23 \%$ & $20,15 \%$ & $15,53 \%$ \\
\hline 8 & 0,5 & $16,01 \%$ & $23,31 \%$ & $35,46 \%$ & $14,05 \%$ & $5,56 \%$ \\
\hline 9 & 0,5 & $0,29 \%$ & $9,06 \%$ & $17,84 \%$ & $31,29 \%$ & $41,52 \%$ \\
\hline
\end{tabular}

Legenda: C.F - Classificação funcional; Recup. - Treinamento de recuperação; Aer. 1 - Zona Aeróbia 1; Aer.2 - Zona Aeróbia 2; Limiar Anae. - Zona de Limiar Anaeróbio; Alta Int. - Zona de Alta Intensidade.

\section{Discussão}

$\mathrm{O}$ valor médio das FC máx. obtidas entre o grupo foi de $138,55 \pm 17,5 \mathrm{bpm}$, o que encontra-se próximo aos achados na literatura. Indivíduos com tetraplegia possuem uma diminuição em sua FC devido à diminuição na estimulação parassimpática vagal (KRASSIOUKOV et al., 2014), a ponto de VALENT (2007) afirmar que, em muitos casos, tetraplégicos possuem uma FC máx. a níveis de apenas 130 bpm. Isso demonstra que, possivelmente, os níveis atingidos durante os jogos foram próximos aos níveis máximos de frequência cardíaca máxima destes indivíduos. No entanto, para confirmar tal possibilidade, seria necessária a realização de testes máximos e uma comparação estatística dos resultados.

A intensidade de jogo encontrada neste estudo foi de $31 \%$ em zona aeróbia 1, 25\% em zona aeróbia 2, 21\% em zona de limiar anaeróbio, $12 \%$ em zona de alta intensidade e $11 \%$ em treinamento de recuperação. Tais valores estão acima dos encontrados na literatura referente à intensidade em termos de velocidade em jogos de RCR. Rhodes et al. (2015a) afirmam que atletas de RCR de modo geral, passam $31 \%$ do tempo de jogo na zona de muito baixa velocidade, $47 \%$ na zona de baixa velocidade, $20 \%$ na zona moderada, $1,5 \%$ na zona alta e $0,5 \%$ na zona de velocidade muito alta.

Essa diferença pode ser explicada por diversas questões, entre elas esta a metodologia, pois, por mais que ambas busquem uma aferição na medição da intensidade, cada uma a fez de um modo diferente, não sendo possível, portanto, uma comparação direta deve ser realizada com cautela. Também pode ser o caso de os atletas monitorados por 
Rodhes et al. (2015) que utlizaram outras táticas e técnicas de jogo (o estudo foi feito na Inglaterra), o que necessariamente faria com que seus tempos de permanência em cada zona de esforço mudassem.

Tais intensidades também não correspondem ao estudo de Santos et al. (2015), em que os atletas permanecem durante a maior parte do tempo, cerca de $54 \%$, em treinamento de recuperação. Porém tal cálculo foi realizado utilizando uma frequência cardíaca máxima predita por equações que não consideram o individuo com LME, o que explicaria tal diferença.

Ao observarmos as intensidades de jogo subdivididas por individuo, foi possível verificar que as mesmas não possuem um padrão aparente, demonstrando uma grande variação em relação à equipe de modo geral, houve grande variação ao se observar cada um dos atletas de cada classe funcional. Tal variação pode ocorrer por diversos motivos, como o fato de que a classificação funcional não levar em consideração o fator autonômico do indivíduo (disfunção autonômica), e também o fato de que o tetraplégico é suscetível a ter disreflexia autonômica, o que pode alterar a sua frequência cardíaca.

Contudo, o presente estudo é de grande relevância devido à carência de estudos com as variáveis estudadas e o RCR, mas acredita-se que novas pesquisas devem ser realizadas em relação a FC máxima, sendo algo limitante em nosso estudo o fato da utilização da linha de corte para a FC máxima atingida pelo atleta e não um teste máximo específico, como uma avaliação de $\mathrm{VO}_{2}$ Máx., o que pode levar a uma diferença.

\section{Conclusão}

Conclui-se que os atletas de RCR apresentaram uma FC máxima reduzida, correspondente às encontradas na literatura, e que os mesmos apresentaram uma variação da intensidade da FC, com predominância entre $60 \%$ a $90 \%$ da FC máxima. Esta é pertinente e esperada, por se tratar de um esporte coletivo que possui como característica a intermitência, além de auxiliar em uma melhor caracterização da modalidade. Tais atletas, por possuírem diferentes níveis de comprometimento fisiológico, acabam por apresentar diferentes variações de níveis de intensidade no decorrer dos jogos.

O estudo demonstrou que o tema abordado deve ser investigado em novos estudos, ultilizando um número maior de atletas de RCR que poderia passar pelos testes máximos, o que permitiria se estabelecer com mais exatidão a FC max. Além de que tais estudos seriam associados com outros fatores de monitoramento de treino, como a percepção subjetiva de esforço, para que se possa obter um resultado mais preciso e uma melhor caracterização do esporte.

\section{Agradecimentos}

Faculdade de Educação Física da Universidade Estadual de Campinas / UNICAMP.

Equipe de rugby em cadeira de rodas ADEACAMP das / ABRC.

\section{Referências}

ABRC - ASSOCIAÇÃO BRASILEIRA DE RUGBY EM

CADEIRA DE RODAS. Disponível em: $<$ http://www.

rugbiabrc.org.br>. Acesso em: 20 Set. 2017.

ARINS, F. B.; SILVA, R. C. R. Intensidade de trabalho durante os treinamentos coletivos de futsal profissional: um estudo de caso. Revista Brasileira de Cineantropometria \& Desempenho Humano. Florianópolis, v. 9, n. 3, p. 291296, 2007.

BORIN, J. P.; PRESTES, J.; MOURA, N. A. Caracterização, Controle e Avaliação: Limitações e Possibilidades no Âmbito do Treinamento Desportivo.

Revista Treinamento Desportivo, v. 8, n. 1, p. 6-11, 2007.

CAMPANA, M. B. et al. O Rugby em Cadeira de Rodas: aspectos técnicos e táticos e diretrizes para seu desenvolvimento. Motriz, Rio Claro, v. 17, n. 4, p. 748-757, out./dez. 2011.

DEVILLARD, X. et al. Effectsof training programs for spinal cordinjury.Annales de Réadaptation et de Médecine Physique, [s.1.], v. 50, n. 6, p. 490-498, jul. 2007.

FLORES, L. J. F. et al. Avaliação da potência aeróbia de praticantes de Rugby em Cadeira de Rodas através de um teste de quadra. Motriz, Rio Claro, v. 19, n. 2, p. 368-377, abr./jun. 2013.

ITURRICASTILLO, A. et al. Objective and subjective methods for quantifying training load in wheelchair basketball small-sided games. Journal of Sports Sciences, [s.1.], p. 1-7, 23 maio 2016a. Informa UK Limited.

ITURRICASTILLO, A. et al. Quantifying Wheelchair Basketball Match Load: A Comparison of Heart-Rate and Perceived-Exertion Methods. International Journal of Sports Physiology And Performance, [s.1.], v. 11, n. 4, p. 508-514, maio 2016b.

IWRF - INTERNATIONAL WHEELCHAIR RUGBY FEDERATION. Disponível em: <http://www.iwrf. com/?page=home $>$. Acesso em 26 Out. 2016.

KRASSIOUKOV, A.; WEST, C. The Role of Autonomic Function on Sport Performance in Athletes With Spinal Cord Injury. Pm\&r. [s. L.], p. 58-65. Aug. 2014.

LAVIS, T. D.; SCELZA, W. M.; BOCKENEK, W. L. Cardiovascular Health and Fitness in Persons with Spinal Cord Injury. Physical Medicine And Rehabilitation Clinics Of North America, [s.1.], v. 18, n. 2, p. 317-331, mai. 2007.

MONTGOMERY, P. G.; PYNE, D. B.; MINAHAN, C. L. The Physical and Physiological Demands of Basketball Training and Competition. International Journal of Sports Physiology And Performance, Australia, v. 5, p.75-86, 2010. 
MYERS, J.; LEE, M.; KIRATLI, J. Cardiovascular Disease in Spinal Cord Injury. American Journal of Physical Medicine \& Rehabilitation, [s.1.], v. 86, n. 2, p. 1-11, fev. 2007.

NASH, M. S. Exercise as a Health-Promoting Activity Following Spinal Cord Injury. Journal of Neurological Physical Therapy, [s. L.], v. 29, n. 2, p. 87-106, 2005.

PAULSON, T. A. W. et al. Individualized Internal and External Training Load Relationships in Elite WheelchairRugby Players. Frontiers In Physiology, [s.1.], v. 6, p. 1-7, 21 dez. 2015.

PELLETIER, C.; HICKS, A. Muscle fatigue characteristics in paralyzed muscle after spinal cord injury. Spinal Cord, Hamilton, v. 49, p. 125-130, 8 June 2011.

SANTOS, R. M.; PENA, L. G.; GORLA, J. I. Análise da Intensidade de Esforço Durante o Jogo de Rugby em Cadeira de Rodas. Revista da Sobama, Marília, v. 16, n. 2 , p. 37-42, Jul./Dez, 2015.

RHODES, J. M. et al. Activity Profiles of Elite Wheelchair Rugby Players during Competition. International Journal of Sports Physiology and Performance, [s.1.], v. 10, n. 3, p. 318-324, abr. 2015a.

RHODES, J. M. et al. Effect of Team Rank and Player Classification on Activity Profiles of Elite Wheelchair Rugby Players. Journal of Sports Sciences, [s.1.], v. 33, n. 19 , p. $2070-2078,27$ mar. 2015 b.

SPORNER, M. L. et al. Quantification of Activity During Wheelchair Basketball and Rugby at the National Veterans Wheelchair Games: A pilot study. Prosthetics And Orthotics International, [s.1.], v. 33, n. 3, p. 210-217, jan. 2009

THOMAS, J. R.; NELSON, J. K.; SILVERMAN, S. J. Métodos de pesquisa em atividade física. 5. ed. Porto Alegre: Artmed, 2007.

VALENT, L. J. M. et al.The individual relationship between heart rate and oxygen uptake in people with a tetraplegia during exercise. Spinal Cord. [s. 1.], p. 104-111. June 2007.

WASHBURN, R. A. et al. The physical activity scale for individuals with physical disabilities: Development and evaluation. Archives of Physical Medicine and Rehabilitation. v. 83, p. 193-200, 2002. 